\title{
FACILE SYNTHESIS AND SPECTROSCOPIC CHARACTERIZATION OF SULFONAMIDE BEARING DIVERSIFIED CARBOXAMIDE AND HYDRAZINE CARBOXAMIDE MOIETIES
}

\author{
Olayinka O. Ajani", Fisayo E. Owolabi, Emmanuel G. Jolayemi, Ifedolapo O. \\ Olanrewaju and Damilola V. Aderohunmu \\ Department of Chemistry, CST, Covenant University, Canaanland, Km 10, Idiroko Road, P.M.B. \\ 1023, Ota, Ogun State, Nigeria. \\ *E-mail: ola.ajani@ covenantuniversity.edu.ng
}

\begin{abstract}
This current research describes the eco-friendly synthesis of $N$-(s-phenyl)-3-phenyl-2-(phenyl sulfonamido) propanamides which are sulfonamide bearing diversified carboxamide moieties. The incorporation of amido functionality into the sulfonamide moieties was herein achieved in three steps in a cost-effective manner by starting from cheap amino acid, phenyl alanine which was reacted with benzenesulfonyl chloride to produce sulfonamide which upon subsequent esterification followed by amidation furnished carboxamido-incorporated sulfonamide analogs 9a-j in good to excellent yield. The completion of reaction processes was authenticated with Thin Layer Chromatography (TLC) and the chemical structures were validated through the elemental analysis result as well as spectroscopic means which include FT-IR, UV, ${ }^{1} \mathrm{H}$ and ${ }^{13} \mathrm{C}$ NMR. The technique used herein was found to be efficient and cost-effective for the production of the series of carboxamide diversified sulfonamide derivatives.
\end{abstract}

Keywords: sulfonamide, spectroscopy, column chromatography, condensation, esterification.

(C) RASĀYAN. All rights reserved

\section{INTRODUCTION}

Sulfonamides are the oldest and remain one of the commonly utilized antimicrobial therapeutics in veterinary medicine, ${ }^{1}$ broadly owing to cost-effectiveness and their relative efficacy in some common bacterial diseases. ${ }^{2}$ Sulfonamide are still widely used for conditions such as acne and urinary tract infections caused by bacteria resistant to other antibiotics. ${ }^{3}$ Sulfonamides constitute a privileged class among pharmacological agents by possessing properties including carbonic anhydrase enzyme (CA) inhibition, as well as diuretic, hypoglycemic, antibacterial, antiviral, and metalloprotease inhibitory effects. ${ }^{4}$ The discovery of valuable antimicrobial properties of azo dye called prontosil by Domagk's paved way for the identification of sulfanilamide as the first efficient agent in therapeutic research. ${ }^{5}$ The systematic review into the past work and usefulness of sulfonamides, unequivocally expresses that apart from the provision of earlier efficient treatment of pathogenic diseases, ${ }^{5,6}$ these class of compounds also unveiled unprecedented revolution in medicine ${ }^{7}$ for the new drug development. ${ }^{8}$ They are preferred due to the ease of administration, ${ }^{9}$ wide spectrum of antimicrobial activity, ${ }^{10}$ noninterference with the host defense mechanism and relative freedom from problems of super-infection. ${ }^{11}$

The biological importance of sulfonamide derivatives is due to their structural resemblance to the naturally occurring $p$-aminobenzoic acid (PABA). The mechanism of action of sulfonamides is well understood to be via competitive inhibitory nature they conferred on $p$-aminobenzoic acid (PABA) thereby resulting in the disruption of the folic acid metabolic pathway and causing inhibition of multiplication of bacteria. ${ }^{12}$ The structural similarity between sulfonamide and para-aminobenzoic acid (PABA) has led to the speculation that it might inhibit dihydropteroate synthase (DHPS) in the folate biosynthetic pathway and cause disruption in the synthesis of tetrahydrofolic acid which is a basic growth factor essential for the 
metabolic process of bacteria. ${ }^{13}$ This allows them to interact with the biopolymers of the living system. ${ }^{14}$ Some commercially available sulfonamide-based drugs that have done healing magic in the therapeutic world are the sulpha drugs 1-6 shown in Fig.-1. Apart from the already marketed drugs, there are many other sulfonamides that are being investigated currently for their promising activity against several malignancies.

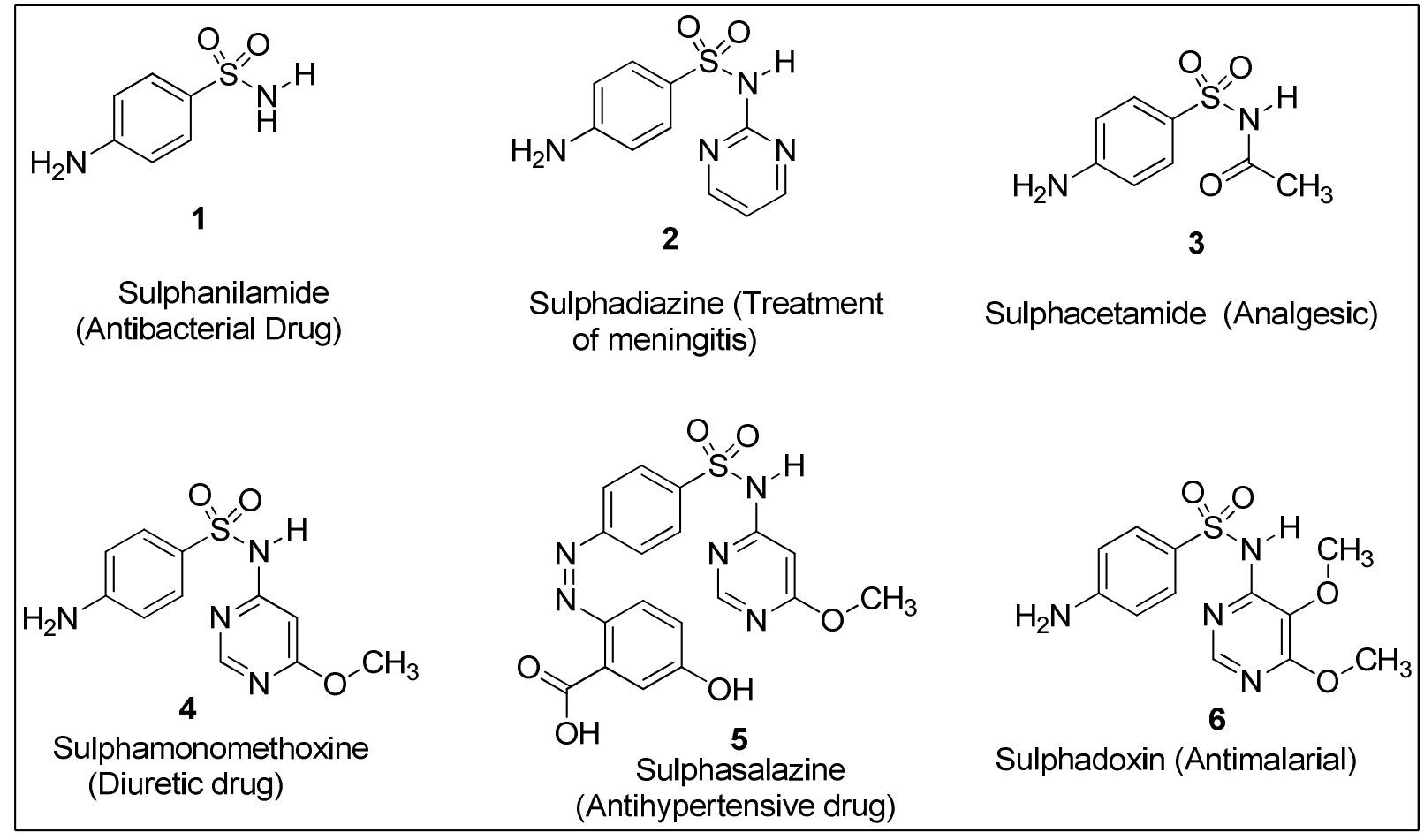

Fig.-1: Selected Commercially available sulfonamide-based drugs

In addition, sulfonamide motif contains a vital functionality owing to its numerous array of documented pharmacological properties which include antimalarial, ${ }^{15}$ antibacterial, ${ }^{16}$ anticancer, ${ }^{17}$ antioxidant, ${ }^{18}$ antitubercular, ${ }^{19}$ anti-HIV, ${ }^{20}$ antitumor, ${ }^{21}$ anticonvulsant, ${ }^{22}$ antidepressant ${ }^{23}$ activities among others. Tolbutamide, the first sulfonylurea anti-diabetic drug, was approved in the United States in 1957 for the treatment of type 2 diabetics. ${ }^{24}$ Similarly, amido containing compounds were known to have highly enhanced pharmacological properties which include, but not limited to including antitubercular, ${ }^{25}$ analgesic, anti-inflammatory, insecticidal, antifungal and antitumor properties. The development of amide is a basic process of high importance in the study of organic compounds. ${ }^{26,27}$ Design of effective methodology for amide preparation stands as an excellent idea because of their significance in basic sciences, leading to many varieties of commercial and medicinal utilization and as crucial synthons in organic synthetic chemistry. ${ }^{26,28}$

Therefore, it was envisaged that inserting of amide group into the sulfonamide moieties might lead to boosting or enhancement of antibacterial activity of such templates. Hence, it is conceivable to design the synthetic route in this present work in such a way to have amide functionality being incorporated within the framework of the synthesized sulfonamides. This might probably lead to the discovery of compounds with increased biological activity for future drug design and help in the comparative study of pharmacological properties of the ordinary sulfonamide to that of amide-bearing sulfonamide derivatives.

\section{EXPERIMENTAL}

All chemical compounds used were purchased from Sigma-Aldrich, USA and British Drug House Chemicals, UK. Solvents used were purified and dried by standard methods where necessary. Melting 
points of solid compounds were established using Stuart melting point machine. The Infrared spectral data were determined with the aid of $\mathrm{KBr}$ pellet using the Perkin Elmer infrared Spectrophotometer and the frequencies of absorption were duly measured in wave number $\left(\mathrm{cm}^{-1}\right)$ from $4000 \mathrm{~cm}^{-1}$ to $500 \mathrm{~cm}^{-1}$. The Ultraviolet-Visible (UV-vis.) spectral data were generated in either dichloromethane $\left(\mathrm{CH}_{2} \mathrm{Cl}_{2}\right)$, or Dimethyl sulphoxide (DMSO) solvents using UV-Genesys Spectrophotometer. The absorbance was plotted against the wavelength $\lambda_{\max }(\mathrm{nm})$ and the obtained molar absorptivity was used to calculate $\log \varepsilon_{\max }$. The reaction completion and degree of purity of the synthesized sulfonamide products were monitored by Thin Layer Chromatography. Furthermore, the ${ }^{1} \mathrm{H}$ NMR and ${ }^{13} \mathrm{C}$ NMR spectral analysis were carried out on NMR Bruker DPX 400 Spectrometer operating at the machine frequencies of $400 \mathrm{MHz}$ and $100 \mathrm{MHz}$ respectively using either $\mathrm{CDCl}_{3}$ or DMSO- $\mathrm{d}_{6}$, as a solvent for sample preparation prior to analyses. The standard abbreviation was used for the multiplicity. The acid value was confirmed with the aid of $\mathrm{pH}$ meter model PHB4. DHG-9023A Vacuum Oven was used to dry the solid where necessary while evaporation of the solvent was done using IKA® RV 10 Rotary evaporator. The determination of \% composition of carbon, hydrogen, and nitrogen of the synthesized sulfonamides was carried out with Flash EA 1112 elemental analyzer. The result of $\%$ found of these elements was in concordance with that of $\%$ calculated values (Table-1).

\section{Synthesis}

\section{Synthesis of 3-phenyl-2-(phenyl sulfonamide)propanoic acid, 7}

$1.113 \mathrm{~g}$ of $\mathrm{Na}_{2} \mathrm{CO}_{3}$ was tipped into a mixture of phenylalanine $(6.00 \mathrm{mmol}, 0.83 \mathrm{~g})$ and distilled water $(6$ $\mathrm{ml}$ ) with continuous stirring at room temperature till complete disappearance of the solute. The mixture placed in an ice bath until the temperature of $10{ }^{\circ} \mathrm{C}$ was attained; then followed by the addition of benzene sulfonyl chloride $(6.00 \mathrm{mmol}, 0.83 \mathrm{ml})$ batch-wisely three times for $1 \mathrm{~h}$ and warmed up to a room temperature also with stirring for $10 \mathrm{~h}$. The reacting solution was worked upon. The first stage, the solution was poured into a separating funnel while the quantity of DCM was added to it, then it was shaking together for $15 \mathrm{mins}$ and then allow to separate. The excess benzene sulfonyl chloride was collected into a $250 \mathrm{ml}$ beaker while the expected product was collected into another big beaker for the better surface area. Secondly, the product collected into a big beaker was also worked upon by adding $2 \mathrm{M} \mathrm{HCl}$ until $\mathrm{pH}$ of 2.1 was reached and the sulfonamide crystallized out immediately. The product was filtered with a filter paper and spread on the beach to dry to afford $91 \%$ yieldo of 7. ${ }^{1} \mathrm{H}-\mathrm{NMR}\left(400 \mathrm{MHz}, \mathrm{DMSOd}_{6}\right) \delta_{\mathrm{H}}$ : 7.61-7.59 (d, $J=8.28 \mathrm{~Hz}, 2 \mathrm{H}, \mathrm{Ar}-\mathrm{H}), 7.24-7.21(\mathrm{~m}, 5 \mathrm{H}, \mathrm{Ar}-\mathrm{H}), 7.10-7.08(\mathrm{~m}, 3 \mathrm{H}, \mathrm{Ar}-\mathrm{H}), 5.16-5.13(\mathrm{~d}, J=8.68 \mathrm{~Hz}, 1 \mathrm{H}$, NH-CH), 4.21-4.17 (m, $1 \mathrm{H}, \mathrm{CH}), 3.12-3.08\left(\mathrm{dd}, J_{1}=5.48 \mathrm{~Hz}, J_{2}=20.00 \mathrm{~Hz}, 1 \mathrm{H}, \mathrm{CH}\right.$ of $\left.\mathrm{CH}_{2}\right), 3.03-2.98$ $\left(\mathrm{dd}, J_{1}=6.40 \mathrm{~Hz}, J_{2}=20.00 \mathrm{~Hz}, 1 \mathrm{H}, \mathrm{CH}\right.$ of $\left.\mathrm{CH}_{2}\right) .{ }^{13} \mathrm{C}-\mathrm{NMR}\left(100 \mathrm{MHz}, \mathrm{DMSOd}_{6}\right) \delta_{\mathrm{C}}: 178.1(\mathrm{C}=\mathrm{O}), 143.9$, 136.6, 134.9, $129.8(2 \times \mathrm{CH}), 129.6(2 \times \mathrm{CH}), 128.8(2 \times \mathrm{CH}), 127.4,127.2(2 \times \mathrm{CH}), 56.5(\mathrm{CH}), 39.0$ $\left(\mathrm{CH}_{2}\right)$ ppm. IR $(\mathrm{KBr}) v_{\max } / \mathrm{cm}^{-1}: 3435(\mathrm{~N}-\mathrm{H}), 3350(\mathrm{OH}), 3050(\mathrm{C}-\mathrm{H}$ aromatic), 2925 (CH aliphatic), 2852 (CH aliphatic), $1730(\mathrm{C}=\mathrm{O}$ of $\mathrm{COOH}), 1605(\mathrm{C}=\mathrm{C}), 1575(\mathrm{C}=\mathrm{N}), 1375\left(\mathrm{SO}_{2}, 1^{\text {st }}\right.$ band $), 1225(\mathrm{C}=\mathrm{N}), 1146$ $\left(\mathrm{SO}_{2}, 2^{\text {nd }}\right.$ band $), 1082(\mathrm{C}=\mathrm{N}), 945(=\mathrm{C}-\mathrm{H}), 724(\mathrm{Ar}-\mathrm{H})$.

\section{Synthesis of ethyl 3-phenyl-2-(phenylsulfonamido)propanoate, 8}

3-Phenyl-2-(phenylsulfonamido)propanoic acid 7 (6.55 mmol, $2.00 \mathrm{~g}$ ) was tipped into $15 \mathrm{ml}$ of absolute $\mathrm{EtOH}$ under continuous stirring at room temperature for about $15 \mathrm{mins}$, followed by the addition of concentrated $\mathrm{H}_{2} \mathrm{SO}_{4}(0.30 \mathrm{ml})$ drop-wisely and stirred at room temperature for additional 15 mins. The resulting solution was refluxed for $2 \mathrm{~h}$ and the solvent was evaporated to dryness with rotary evaporator. The resulting solid was triturated with cold water. It was filtered and air-dried to obtain 3-phenyl-2(phenylsulfonamido)propanoate, 8 in $66 \%$ yield. ${ }^{1} \mathrm{H}-\mathrm{NMR}\left(400 \mathrm{MHz}, \mathrm{DMSOd}_{6}\right) \delta_{\mathrm{H}}: 7.63-7.61(\mathrm{~d}, J=8.32$ $\mathrm{Hz}, 2 \mathrm{H}, \mathrm{Ar}-\mathrm{H}), 7.25-7.18$ (m, 5H, Ar-H), 7.13-7.10 (m, 3H, Ar-H), 5.88-5.86 (d, J = 9.44 Hz, 1H, NH-CH), 4.31-4.25 (m, $1 \mathrm{H}, \mathrm{CH}), 3.13-3.09\left(\mathrm{dd}, J_{1}=5.48 \mathrm{~Hz}, J_{2}=20.00 \mathrm{~Hz}, 1 \mathrm{H}, \mathrm{CH}\right.$ of $\left.\mathrm{CH}_{2}\right), 3.04-2.99\left(\mathrm{dd}, J_{1}=\right.$ $6.40 \mathrm{~Hz}, J_{2}=20.00 \mathrm{~Hz}, 1 \mathrm{H}, \mathrm{CH}$ of $\left.\mathrm{CH}_{2}\right), 2.79-2.74\left(\mathrm{q}, J=7.12 \mathrm{~Hz}, 2 \mathrm{H}, \underline{\mathrm{CH}_{2}}-\mathrm{CH}_{3}\right), 0.88-0.84$ (t, $J=7.12$ $\left.\mathrm{Hz}, 3 \mathrm{H}, \mathrm{CH}_{3}-\mathrm{CH}_{2}\right) .{ }^{13} \mathrm{C}-\mathrm{NMR}\left(100 \mathrm{MHz}, \mathrm{DMSOd}_{6}\right) \delta_{\mathrm{C}}: 175.7(\mathrm{C}=\mathrm{O}), 143.1,136.6,134.8,129.9(2 \times \mathrm{CH})$, $129.5(2 \times \mathrm{CH}), 128.8(2 \times \mathrm{CH}), 127.5,127.1(2 \times \mathrm{CH}), 56.7(\mathrm{CH}), 42.5\left(\mathrm{CH}_{2}\right), 39.3\left(\mathrm{CH}_{2}\right), 19.2\left(\mathrm{CH}_{3}\right)$ ppm. IR (KBr) $v_{\max } / \mathrm{cm}^{-1}: 3435(\mathrm{~N}-\mathrm{H}), 3042(\mathrm{C}-\mathrm{H}$ aromatic), 2925 (CH aliphatic), 2852 (CH aliphatic), 
$1745(\mathrm{C}=\mathrm{O}$ of ester $), 1605(\mathrm{C}=\mathrm{C}), 1574(\mathrm{C}=\mathrm{N}), 1452\left(\mathrm{CH}_{2}\right.$ deformation $), 1377\left(\mathrm{SO}_{2}, 1^{\text {st }}\right.$ band $), 1225(\mathrm{C}=\mathrm{N})$, $1146\left(\mathrm{SO}_{2}, 2^{\text {nd }}\right.$ band $), 1082(\mathrm{C}-\mathrm{N}), 946(=\mathrm{C}-\mathrm{H}), 725(\mathrm{Ar}-\mathrm{H})$.

\section{General procedure for synthesis of targeted sulfonamide derivatives, 9a-j}

Ethyl 3-phenyl-2-(phenyl sulfonamide)propanoate, $8(3.30 \mathrm{mmol}, 1.00 \mathrm{~g})$, was transferred into $250 \mathrm{ml}$ quick fit flask containing absolute EtOH $(15 \mathrm{ml})$; then the resulting solution was allowed to stir at ambient temperature for $15 \mathrm{mins}$. The corresponding amino-containing nucleophile $(3.30 \mathrm{mmol})$ was weighed and transferred into the solution and stirred for extra $15 \mathrm{mins}$ at the same ambient condition. The reacting solution was then refluxed for $2 \mathrm{~h}$ and the solvent was evaporated to afford crude solids which were recrystallized from methanol to furnish the corresponding amido-bearing sulfonamide motifs 9a-j in varying yields.

\section{Synthesis of $N, 3$-diphenyl-2-(phenyl sulfonamide)propanamide, 9a}

Reactive intermediate $8(3.30 \mathrm{mmol}, 1.00 \mathrm{~g})$ reacted with aniline $(3.30 \mathrm{mmol}, 0.30 \mathrm{ml})$ to afford $N, 3$ diphenyl-2-(phenylsulfonamido)propanamide, 9a in 86\% yield. ${ }^{1} \mathrm{H}-\mathrm{NMR}\left(400 \mathrm{MHz}, \mathrm{DMSOd}_{6}\right) \delta_{\mathrm{H}}: 11.08$ (s, $1 \mathrm{H}, \underline{\mathrm{NH}}-\mathrm{CO}), 7.62-7.60(\mathrm{~d}, J=8.34 \mathrm{~Hz}, 2 \mathrm{H}, \mathrm{Ar}-\mathrm{H}), 7.45-7.43(\mathrm{~d}, J=8.00 \mathrm{~Hz}, 2 \mathrm{H}, \mathrm{Ar}-\mathrm{H}), 7.27-7.24(\mathrm{~m}$, 5H, Ar-H), 7.20-7.18 (m, 3H, Ar-H), 7.15-7.12 (m, 3H, Ar-H), 5.88-5.86 (d, J = 9.42 Hz, 1H, NH-CH), 4.31-4.25 $(\mathrm{m}, 1 \mathrm{H}, \mathrm{CH}), 3.13-3.09\left(\mathrm{dd}, J_{1}=5.48 \mathrm{~Hz}, J_{2}=20.00 \mathrm{~Hz}, 1 \mathrm{H}, \mathrm{CH}\right.$ of $\left.\mathrm{CH}_{2}\right), 3.04-2.99\left(\mathrm{dd}, J_{1}=\right.$ $6.40 \mathrm{~Hz}, J_{2}=20.00 \mathrm{~Hz}, 1 \mathrm{H}, \mathrm{CH}$ of $\left.\mathrm{CH}_{2}\right) .{ }^{13} \mathrm{C}-\mathrm{NMR}\left(100 \mathrm{MHz}, \mathrm{DMSOd}_{6}\right) \delta_{\mathrm{C}}: 171.3(\mathrm{C}=\mathrm{O}), 145.1,143.8$, 136.7, 134.9, $130.2(2 \times \mathrm{CH}), 129.8(2 \times \mathrm{CH}), 129.6(2 \times \mathrm{CH}), 128.8(2 \times \mathrm{CH}), 127.4,127.2(2 \times \mathrm{CH})$, 119.1, $115.2(2 \times \mathrm{CH}), 56.5(\mathrm{CH}), 39.0\left(\mathrm{CH}_{2}\right)$ ppm. IR $(\mathrm{KBr}) v_{\max } / \mathrm{cm}^{-1}: 3415(\mathrm{~N}-\mathrm{H}), 2930(\mathrm{C}-\mathrm{H}$ aliphatic), 2850 (C-H aliphatic), $1685(\mathrm{C}=\mathrm{O}), 1610\left(\mathrm{C}=\mathrm{C}\right.$ aromatic), $1491(\mathrm{~N}-\mathrm{H}$ amide $), 1459\left(\mathrm{CH}_{2}\right.$ deformation), $1377\left(\mathrm{SO}_{2}, 1^{\text {st }}\right.$ band $), 1140\left(\mathrm{SO}_{2}, 2^{\text {nd }}\right.$ band $), 1073(\mathrm{C}-\mathrm{N}), 952(=\mathrm{C}-\mathrm{H}), 848(\mathrm{C}=\mathrm{C}$ out of plane bending $), 743$ (Ar-H, bending and ring puckering). $\lambda_{\max } / \mathrm{nm}$ (log $\left.\varepsilon_{\max }\right): 207$ (5.01), 244 (5.47), 286 (5.11), 583 (2.00).

\section{Synthesis of $N$-(3-nitrophenyl)-3-phenyl-2-(phenylsulfonamido)propanamide, $9 \mathrm{~b}$}

Reactive intermediate $8(3.30 \mathrm{mmol}, 1.00 \mathrm{~g})$ reacted with 3-nitroaniline $(3.30 \mathrm{mmol}, 0.45 \mathrm{~g})$ to afford $\mathrm{N}$ (3-nitrophenyl)-3-phenyl-2-(phenylsulfonamido) propanamide, $9 \mathbf{b}$ in $87 \%$ yield. ${ }^{1} \mathrm{H}-\mathrm{NMR}$ (400 $\mathrm{MHz}$, $\left.\mathrm{DMSOd}_{6}\right) \delta_{\mathrm{H}}: 11.10(\mathrm{~s}, 1 \mathrm{H}, \underline{\mathrm{NH}}-\mathrm{CO}), 8.01(\mathrm{~s}, 1 \mathrm{H}, \mathrm{Ar}-\mathrm{H}), 7.62-7.60(\mathrm{~d}, J=8.34 \mathrm{~Hz}, 2 \mathrm{H}, \mathrm{Ar}-\mathrm{H}), 7.47-7.46$ $(\mathrm{d}, J=6.20 \mathrm{~Hz}, 1 \mathrm{H}, \mathrm{Ar}-\mathrm{H}), 7.27-7.24$ (m, 5H, Ar-H), 7.20-7.18 (m, 3H, Ar-H), 7.14-7.12 (m, 2H, Ar-H), $5.87-5.84(\mathrm{~d}, J=9.04 \mathrm{~Hz}, 1 \mathrm{H}, \underline{\mathrm{NH}}-\mathrm{CH}), 4.31-4.25(\mathrm{~m}, 1 \mathrm{H}, \mathrm{CH}), 3.14-3.10\left(\mathrm{dd}, J_{1}=5.86 \mathrm{~Hz}, J_{2}=20.00\right.$ $\mathrm{Hz}, 1 \mathrm{H}, \mathrm{CH}$ of $\left.\mathrm{CH}_{2}\right), 3.04-2.99\left(\mathrm{dd}, J_{1}=6.44 \mathrm{~Hz}, J_{2}=20.00 \mathrm{~Hz}, 1 \mathrm{H}, \mathrm{CH}\right.$ of $\left.\mathrm{CH}_{2}\right) .{ }^{13} \mathrm{C}-\mathrm{NMR}(100 \mathrm{MHz}$, $\left.\mathrm{DMSOd}_{6}\right) \delta_{\mathrm{C}}: 171.3(\mathrm{C}=\mathrm{O}), 145.1,144.2,143.8,136.7,134.9,130.2(2 \times \mathrm{CH}), 129.8(2 \times \mathrm{CH}), 129.6(2 \times$ $\mathrm{CH}), 128.8(2 \times \mathrm{CH}), 127.4,127.2(2 \times \mathrm{CH}), 119.1,115.3,56.4(\mathrm{CH}), 39.1\left(\mathrm{CH}_{2}\right) \mathrm{ppm}$. IR $(\mathrm{KBr}) v_{\max } / \mathrm{cm}^{-}$ 1: $3422(\mathrm{~N}-\mathrm{H}), 3029$ (C-H aromatic), 2923 (C-H aliphatic), 2853 (C-H aliphatic), 1685 (C=O amide), 1600 $(\mathrm{C}=\mathrm{C}$ aromatic $), 1579(\mathrm{C}=\mathrm{N}), 1459\left(\mathrm{CH}_{2}\right.$ deformation $), 1420\left(\mathrm{CH}_{2}\right.$ deformation $), 1377\left(\mathrm{SO}_{2}, 1^{\text {st }}\right.$ band $)$, $1352(\mathrm{~N}=\mathrm{O}$ stretching $), 1228(\mathrm{C}-\mathrm{N}), 1127\left(\mathrm{SO}_{2}, 1^{\text {st }}\right.$ band $), 921(=\mathrm{C}-\mathrm{H}), 735(\mathrm{Ar}-\mathrm{H}) \cdot \lambda_{\max } / \mathrm{nm}\left(\log \varepsilon_{\max }\right): 206$ (4.72), 227 (4.20), 251 (3.99), 374 (4.23).

\section{Synthesis of $N$-(4-chlorophenyl)-3-phenyl-2-(phenylsulfonamido)propanamide, 9c}

Reactive intermediate $8(3.30 \mathrm{mmol}, 1.00 \mathrm{~g})$ reacted with 4-chloroaniline $(3.30 \mathrm{mmol}, 0.42 \mathrm{~g})$ to afford $N$ (4-chlorophenyl)-3-phenyl-2-(phenylsulfonamido)propanamide, 9c in 74\% yield. ${ }^{1} \mathrm{H}-\mathrm{NMR}$ (400 $\mathrm{MHz}$, $\left.\mathrm{DMSOd}_{6}\right) \delta_{\mathrm{H}}: 11.04(\mathrm{~s}, 1 \mathrm{H}, \underline{\mathrm{NH}}-\mathrm{CO}), 7.62-7.60(\mathrm{~d}, J=8.26 \mathrm{~Hz}, 2 \mathrm{H}, \mathrm{Ar}-\mathrm{H}), 7.42-7.40$ (d, $J=8.00 \mathrm{~Hz}, 2 \mathrm{H}$, Ar-H), 7.25-7.21 (m, 5H, Ar-H), 7.16-7.14 (d, $J=8.12 \mathrm{~Hz}, 2 \mathrm{H}, \mathrm{Ar}-\mathrm{H}), 7.10-7.07$ (m, 3H, Ar-H), 5.16-5.14 $(\mathrm{d}, J=7.68 \mathrm{~Hz}, 1 \mathrm{H}, \underline{\mathrm{NH}}-\mathrm{CH}), 4.20-4.17(\mathrm{~m}, 1 \mathrm{H}, \mathrm{CH}), 3.12-3.07\left(\mathrm{dd}, J_{1}=6.02 \mathrm{~Hz}, J_{2}=20.00 \mathrm{~Hz}, 1 \mathrm{H}, \mathrm{CH}\right.$ of $\left.\mathrm{CH}_{2}\right), 3.03-2.98\left(\mathrm{dd}, J_{1}=6.40 \mathrm{~Hz}, J_{2}=20.00 \mathrm{~Hz}, 1 \mathrm{H}, \mathrm{CH}\right.$ of $\left.\mathrm{CH}_{2}\right) .{ }^{13} \mathrm{C}-\mathrm{NMR}\left(100 \mathrm{MHz}, \mathrm{DMSOd}_{6}\right) \delta_{\mathrm{C}}$ : $171.3(\mathrm{C}=\mathrm{O}), 145.1,143.9,142.7,136.6,134.9,129.8(2 \times \mathrm{CH}), 129.6(2 \times \mathrm{CH}), 128.8(2 \times \mathrm{CH}), 127.4$, $127.2(2 \times \mathrm{CH}), 123.7(2 \times \mathrm{CH}), 115.2(2 \times \mathrm{CH}), 56.7(\mathrm{CH}), 39.2\left(\mathrm{CH}_{2}\right) \mathrm{ppm}$. IR $(\mathrm{KBr}) v_{\max } / \mathrm{cm}^{-1}: 3350(\mathrm{~N}-$ $\mathrm{H}, 2^{\circ}$ amine), 3100 (C-H aromatic), 2925 (C-H aliphatic), 2852 (C-H aliphatic), 1685 (C=O amide), 1620 $(\mathrm{C}=\mathrm{C}$ aromatic $), 1599(\mathrm{C}=\mathrm{N}), 1496\left(\mathrm{CH}_{2}\right.$, deformation $), 1377\left(\mathrm{SO}_{2}, 1^{\text {st }}\right.$ band $), 1220(\mathrm{C}=\mathrm{N}), 1130\left(\mathrm{SO}_{2} 2^{\text {nd }}\right.$ 
RASĀYAN J. Chem.

Vol. 10 | No. 4 |1402-1411 | October - December | 2017

band), 1074 (C-N), 745 (Ar-H), 698 (C-Cl bending). $\lambda_{\max } / \mathrm{nm}$ (log $\left.\varepsilon_{\max }\right): 207$ (4.71), 251 (3.70), 269 (3.64), 323 (4.14).

Synthesis of $N$-(naphthalen-1-yl)-3-phenyl-2-(phenylsulfonamido)propanamide, 9d

Reactive intermediate $8(3.30 \mathrm{mmol}, 1.00 \mathrm{~g})$ reacted with $\alpha$-naphthylamine $(3.30 \mathrm{mmol}, 0.47 \mathrm{~g})$ to afford $N$-(naphthalen-1-yl)-3-phenyl-2-(phenylsulfonamido)propanamide, 9d in $65 \%$ yield. ${ }^{1} \mathrm{H}-\mathrm{NMR}(400 \mathrm{MHz}$, $\left.\mathrm{DMSOd}_{6}\right) \delta_{\mathrm{H}}: 11.05(\mathrm{~s}, 1 \mathrm{H}, \underline{\mathrm{NH}}-\mathrm{CO}), 8.16-8.14(\mathrm{~d}, J=7.24 \mathrm{~Hz}, 1 \mathrm{H}, \mathrm{Ar}-\mathrm{H}), 8.04-8.02(\mathrm{~d}, J=7.66 \mathrm{~Hz}, 1 \mathrm{H}$, Ar-H), 7.61-7.59 (d, $J=8.28 \mathrm{~Hz}, 2 \mathrm{H}, \mathrm{Ar}-\mathrm{H}), 7.57-7.53(\mathrm{~m}, 3 \mathrm{H}, \mathrm{Ar}-\mathrm{H}), 733-7.32(\mathrm{~d}, J=7.04 \mathrm{~Hz}, 1 \mathrm{H}, \mathrm{Ar}-$ H), 7.24-7.21 (m, 5H, Ar-H), 7.17-7.15 (d, J= 7.14 Hz, 1H, Ar-H), 7.10-7.08 (m, 3H, Ar-H), 5.16-5.13 (d, $J=8.68 \mathrm{~Hz}, 1 \mathrm{H}, \underline{\mathrm{NH}}-\mathrm{CH}), 4.21-4.18(\mathrm{~m}, 1 \mathrm{H}, \mathrm{CH}), 3.11-3.08\left(\mathrm{dd}, J_{1}=5.50 \mathrm{~Hz}, J_{2}=20.00 \mathrm{~Hz}, 1 \mathrm{H}, \mathrm{CH}\right.$ of $\left.\mathrm{CH}_{2}\right), 3.02-2.98\left(\mathrm{dd}, J_{1}=6.38 \mathrm{~Hz}, J_{2}=20.00 \mathrm{~Hz}, 1 \mathrm{H}, \mathrm{CH}\right.$ of $\left.\mathrm{CH}_{2}\right) \cdot{ }^{13} \mathrm{C}-\mathrm{NMR}\left(100 \mathrm{MHz}, \mathrm{DMSOd}_{6}\right) \delta_{\mathrm{C}}$ : $171.0(\mathrm{C}=\mathrm{O}), 145.3,143.1,139.8,136.6,134.8,133.2,132.4,130.1,129.9(2 \times \mathrm{CH}), 129.5(2 \times \mathrm{CH}), 128.8$ $(2 \times \mathrm{CH}), 127.5,127.1(2 \times \mathrm{CH}), 125.7,123.4,119.9,119.2,115.2,56.7(\mathrm{CH}), 39.3\left(\mathrm{CH}_{2}\right) \mathrm{ppm} . \mathrm{IR}(\mathrm{KBr})$ $v_{\max } / \mathrm{cm}^{-1}: 3400(\mathrm{~N}-\mathrm{H}), 3045(\mathrm{CH}$ aromatic $), 2924(\mathrm{CH}$ aliphatic $), 2854(\mathrm{C}-\mathrm{H}$ aliphatic $), 1605(\mathrm{C}=\mathrm{C}$ aromatic), $1555(\mathrm{C}=\mathrm{N}), 1511\left(\mathrm{~N}-\mathrm{H} 2^{\circ}\right.$ amine $), 1461\left(\mathrm{CH}_{2}\right.$ deformation $), 1377\left(\mathrm{SO}_{2} 1^{\text {st }}\right.$ band $), 1216(\mathrm{C}=\mathrm{N})$, $1130\left(\mathrm{SO}_{2} 2^{\text {nd }}\right.$ band), $1073(\mathrm{C}-\mathrm{N}), 912(=\mathrm{C}-\mathrm{H}), 766(\mathrm{Ar}-\mathrm{H}) \cdot \lambda_{\max } / \mathrm{nm}\left(\log \varepsilon_{\max }\right): 209(4.69), 269(4.16), 332$ (5.43), 578 (3.23).

\section{Synthesis of $\boldsymbol{N}$-(1-hydrazinyl-1-oxo-3-phenylpropane-2-yl) benzenesulfonamide, $9 \mathrm{e}$}

Reactive intermediate $8(3.30 \mathrm{mmol}, 1.00 \mathrm{~g})$ reacted with hydrazine hydrate $(3.30 \mathrm{mmol}, 0.16 \mathrm{ml})$ to afford $\mathrm{N}$-(1-hydrazinyl-1-oxo-3-phenylpropan-2-yl)benzenesulfonamide, $9 \mathrm{e}$ in $54 \%$ yield. ${ }^{1} \mathrm{H}-\mathrm{NMR}$ (400 MHz, $\left.\mathrm{DMSOd}_{6}\right) \delta_{\mathrm{H}}: 11.00$ (s, 1H, NH-CO), 7.62-7.60 (d, $\left.J=8.32 \mathrm{~Hz}, 2 \mathrm{H}, \mathrm{Ar}-\mathrm{H}\right), 7.24-7.21(\mathrm{~m}, 5 \mathrm{H}, \mathrm{Ar}-\mathrm{H}), 7.10-$ $7.08(\mathrm{~m}, 3 \mathrm{H}, \mathrm{Ar}-\mathrm{H}), 5.40\left(\mathrm{~s}, 2 \mathrm{H}, \mathrm{NH}_{2}\right), 5.14-5.11(\mathrm{~d}, J=8.60 \mathrm{~Hz}, 1 \mathrm{H}, \underline{\mathrm{NH}}-\mathrm{CH}), 4.20-4.17(\mathrm{~m}, 1 \mathrm{H}, \mathrm{CH})$, $3.13-3.08\left(\mathrm{dd}, J_{1}=5.82 \mathrm{~Hz}, J_{2}=20.00 \mathrm{~Hz}, 1 \mathrm{H}, \mathrm{CH}\right.$ of $\left.\mathrm{CH}_{2}\right), 3.02-2.97 \overline{(\mathrm{dd}}, J_{1}=6.44 \mathrm{~Hz}, J_{2}=20.00 \mathrm{~Hz}$, $1 \mathrm{H}, \mathrm{CH}$ of $\left.\mathrm{CH}_{2}\right) .{ }^{13} \mathrm{C}-\mathrm{NMR}\left(100 \mathrm{MHz}, \mathrm{DMSOd}_{6}\right) \delta_{\mathrm{C}}: 172.1(\mathrm{C}=\mathrm{O}), 143.9,136.3,134.7,129.9(2 \times \mathrm{CH})$, $129.4(2 \times \mathrm{CH}), 128.8(2 \times \mathrm{CH}), 127.4,127.1(2 \times \mathrm{CH}), 56.4(\mathrm{CH}), 39.1\left(\mathrm{CH}_{2}\right) \mathrm{ppm}$. IR $(\mathrm{KBr}) v_{\max } / \mathrm{cm}^{-1}$ : $3450(\mathrm{~N}-\mathrm{H}), 3100$ (CH aromatic), 2924, 2854 (C-H aliphatic), 1627 (C=C aromatic), $1560(\mathrm{C}=\mathrm{N}), 1459$ $\left(\mathrm{CH}_{2}\right.$ deformation $), 1377\left(\mathrm{SO}_{2} 1^{\text {st }}\right.$ band $), 1226(\mathrm{C}=\mathrm{N}), 1129\left(\mathrm{SO}_{2} 2^{\text {nd }}\right.$ band $), 1074(\mathrm{C}-\mathrm{N}), 913(=\mathrm{C}-\mathrm{H}), 746$ (Ar-H). $\lambda_{\max } / \mathrm{nm}\left(\log \varepsilon_{\max }\right): 206$ (4.57), 242 (4.33), 545 (4.32), 584 (4.34).

\section{Synthesis of $\quad N$-(1-(2-(2,4-dinitrophenyl)hydrazinyl)-1-oxo-3-phenylpropan-2-yl)benzene sulfonamide, $9 \mathrm{f}$}

Reactive intermediate $8(3.30 \mathrm{mmol}, 1.00 \mathrm{~g})$ reacted with 2,4-dinitrophenylhydrazine (3.30 mmol, $0.65 \mathrm{~g})$ to afford $N$-(1-(2-(2,4-dinitrophenyl)hydrazinyl)-1-oxo-3-phenylpropan-2-yl) benzene sulfonamide, 9f in $82 \%$ yield. ${ }^{1} \mathrm{H}-\mathrm{NMR}\left(400 \mathrm{MHz}, \mathrm{DMSOd}_{6}\right) \delta_{\mathrm{H}}: 11.05$ (s, 1H, NH-CO), 10.00 (s, 1H, NH), 8.82 (s, 1H, ArH), 828-8.25 (d, $J=12.36 \mathrm{~Hz}, 1 \mathrm{H}, \mathrm{Ar}-\mathrm{H}), 7.69-7.66(\mathrm{~d}, J=9.72 \mathrm{~Hz}, 1 \mathrm{H}, \mathrm{Ar}-\mathrm{H}), 7.31-7.25(\mathrm{~m}, 6 \mathrm{H}, \mathrm{Ar}-\mathrm{H})$, $7.00(\mathrm{~s}, 5 \mathrm{H}, \mathrm{Ar}-\mathrm{H}), 5.20(\mathrm{~s}, 1 \mathrm{H}, \mathrm{NH}), 3.92(\mathrm{~m}, 1 \mathrm{H}, \mathrm{CH}), 3.12-3.07\left(\mathrm{dd}, J_{1}=6.02 \mathrm{~Hz}, J_{2}=20.00 \mathrm{~Hz}, 1 \mathrm{H}\right.$, $\mathrm{CH}$ of $\left.\mathrm{CH}_{2}\right), 3.03-2.98\left(\mathrm{dd}, J_{1}=6.40 \mathrm{~Hz}, J_{2}=20.00 \mathrm{~Hz}, 1 \mathrm{H}, \mathrm{CH}\right.$ of $\left.\mathrm{CH}_{2}\right) .{ }^{13} \mathrm{C}-\mathrm{NMR}\left(100 \mathrm{MHz}, \mathrm{DMSOd}_{6}\right)$ $\delta_{\mathrm{C}}: 171.3(\mathrm{C}=\mathrm{O}), 143.1,141.7,139.7,139.1,136.6,134.8,129.9(2 \times \mathrm{CH}), 129.3(2 \times \mathrm{CH}), 128.7(2 \times \mathrm{CH})$, 127.5, $127.1(2 \times \mathrm{CH}), 125.7,123.2,119.6,56.8(\mathrm{CH}), 39.1\left(\mathrm{CH}_{2}\right) \mathrm{ppm}$. IR $(\mathrm{KBr}) v_{\max } / \mathrm{cm}^{-1}: 3367(\mathrm{~N}-\mathrm{H})$, $2924\left(\mathrm{C}-\mathrm{H}\right.$ of $\left.\mathrm{CH}_{2}\right), 1676(\mathrm{C}=\mathrm{O}$ of amide $), 1601(\mathrm{C}=\mathrm{C}), 1489\left(\mathrm{CH}_{2}\right.$ deformation $), 1376\left(\mathrm{SO}_{2}\right), 1182\left(\mathrm{SO}_{2}\right.$, $2^{\text {nd }}$ band), $1092(\mathrm{C}-\mathrm{N}), 980$ (=C-H) $747(\mathrm{Ar}-\mathrm{H}) . \lambda_{\max } / \mathrm{nm}\left(\log \varepsilon_{\max }\right): 209$ (4.83), 254 (4.73), 278 (4.75), 362 (5.46).

\section{Synthesis of 2-(3-phenyl-2-(phenyl sulfonamide)propanoyl)hydrazinecarboxamide, 9g}

Reactive intermediate $8(3.30 \mathrm{mmol}, 1.00 \mathrm{~g})$ reacted with semicarbazide $(3.30 \mathrm{mmol}, 0.25 \mathrm{~g})$ to afford 2(3-phenyl-2-(phenylsulfonamido)propanoyl)hydrazinecarboxamide, $9 \mathrm{~g}$ in $55 \%$ yield. ${ }^{1} \mathrm{H}-\mathrm{NMR}(400 \mathrm{MHz}$, $\left.\mathrm{DMSOd}_{6}\right) \delta_{\mathrm{H}}: 11.05(\mathrm{~s}, 1 \mathrm{H}, \mathrm{NH}-\mathrm{CO}), 10.54(\mathrm{~s}, 1 \mathrm{H}, \mathrm{NH}), 8.41\left(\mathrm{~s}, 1 \mathrm{H}, \mathrm{NH}\right.$ of $\left.\mathrm{NH}_{2}\right), 7.95(\mathrm{~s}, 1 \mathrm{H}, \mathrm{NH} \text { of NH})_{2}$, 7.61-7.59 (d, $J=8.30 \mathrm{~Hz}, 2 \mathrm{H}$, Ar-H), 7.24-7.20 (m, 5H, Ar-H), 7.11-7.08 (m, 3H, Ar-H), 5.13-5.11 (d, $J=$ $7.86 \mathrm{~Hz}, 1 \mathrm{H}, \underline{\mathrm{NH}}-\mathrm{CH}), 4.20-4.16(\mathrm{~m}, 1 \mathrm{H}, \mathrm{CH}), 3.13-3.08\left(\mathrm{dd}, J_{1}=5.82 \mathrm{~Hz}, J_{2}=20.00 \mathrm{~Hz}, 1 \mathrm{H}, \mathrm{CH}\right.$ of $\left.\mathrm{CH}_{2}\right)$, 3.02-2.98 (dd, $J_{1}=6.40 \mathrm{~Hz}, J_{2}=20.00 \mathrm{~Hz}, 1 \mathrm{H}, \mathrm{CH}$ of $\left.\mathrm{CH}_{2}\right) .{ }^{13} \mathrm{C}-\mathrm{NMR}\left(100 \mathrm{MHz}, \mathrm{DMSOd}_{6}\right) \delta_{\mathrm{C}}: 172.9$ 
RASĀYAN $J$. Chem.

Vol. 10 | No. 4 |1402-1411 | October - December | 2017

$(\mathrm{C}=\mathrm{O}), 172.1(\mathrm{C}=\mathrm{O}), 143.9,136.3,134.7,129.9(2 \times \mathrm{CH}), 129.4(2 \times \mathrm{CH}), 128.8(2 \times \mathrm{CH}), 127.4,127.1$ $(2 \times \mathrm{CH}), 56.2(\mathrm{CH}), 39.4\left(\mathrm{CH}_{2}\right) \mathrm{ppm}$. IR $(\mathrm{KBr}) v_{\max } / \mathrm{cm}^{-1}: 3422,3419\left(\mathrm{NH}_{2}, 2\right.$ bands $), 3367(\mathrm{~N}-\mathrm{H}), 3035$ ( $\mathrm{CH}$ aromatic), $2928\left(\mathrm{C}-\mathrm{H}\right.$ of $\left.\mathrm{CH}_{2}\right), 1687(\mathrm{C}=\mathrm{O}$ of amide $), 1676(\mathrm{C}=\mathrm{O}$ of amide $), 1615(\mathrm{C}=\mathrm{C}), 1575(\mathrm{C}=\mathrm{N})$, $1485\left(\mathrm{CH}_{2}\right.$ deformation), $1375\left(\mathrm{SO}_{2}\right), 1175\left(\mathrm{SO}_{2}, 2^{\text {nd }}\right.$ band $), 1095(\mathrm{C}-\mathrm{N}), 965(=\mathrm{C}-\mathrm{H}), 745(\mathrm{Ar}-\mathrm{H}) . \lambda_{\max } / \mathrm{nm}$ (log $\left.\varepsilon_{\max }\right): 212$ (4.73), 230 (3.68), 251 (3.92), 329 (3.59).

\section{Synthesis of $N$-pentyl-3-phenyl-2-(phenylsulfonamido)propanamide, $9 \mathrm{~h}$}

Reactive intermediate $8(3.30 \mathrm{mmol}, 1.00 \mathrm{~g})$ reacted with $n$-pentylamine $(3.30 \mathrm{mmol}, 0.43 \mathrm{ml})$ to afford $N$ pentyl-3-phenyl-2-(phenylsulfonamido)propanamide, $9 \mathbf{h}$ in $89 \%$ yield. ${ }^{1} \mathrm{H}-\mathrm{NMR}\left(400 \mathrm{MHz}, \mathrm{DMSOd}_{6}\right) \delta_{\mathrm{H}}$ : 7.61-7.59 (d, $J=8.28 \mathrm{~Hz}, 2 \mathrm{H}, \mathrm{Ar}-\mathrm{H}), 7.24-7.21(\mathrm{~m}, 5 \mathrm{H}, \mathrm{Ar}-\mathrm{H}), 7.10-7.08(\mathrm{~m}, 3 \mathrm{H}, \mathrm{Ar}-\mathrm{H}), 5.89-5.87(\mathrm{~d}, J=$ $8.88 \mathrm{~Hz}, 1 \mathrm{H}, \underline{\mathrm{NH}}-\mathrm{CH}), 4.30-4.26(\mathrm{~m}, 1 \mathrm{H}, \mathrm{CH}), 3.44-3.43\left(\mathrm{t}, J=3.28 \mathrm{~Hz}, 2 \mathrm{H}, \underline{\mathrm{NCH}_{2}} \mathrm{CH}_{2}\right), 1.96-1.93(\mathrm{~m}$, $\left.4 \mathrm{H}, 2 \times \mathrm{CH}_{2}\right), 1.49-1.46\left(\mathrm{~m}, 2 \mathrm{H}, \mathrm{CH}_{2}\right), 3.12-3.08\left(\mathrm{dd}, J_{1}=5.48 \mathrm{~Hz}, J_{2}=20.00 \mathrm{~Hz}, 1 \mathrm{H}, \mathrm{CH}\right.$ of $\left.\mathrm{CH}_{2}\right), 3.03-$ $2.98\left(\mathrm{dd}, J_{1}=6.40 \mathrm{~Hz}, J_{2}=20.00 \mathrm{~Hz}, 1 \mathrm{H}, \mathrm{CH}\right.$ of $\left.\mathrm{CH}_{2}\right), 0.90-0.88\left(\mathrm{t}, J=3.96 \mathrm{~Hz}, 2 \mathrm{H}, \underline{\mathrm{CH}}_{3} \mathrm{CH}_{2}\right) .{ }^{13} \mathrm{C}-\mathrm{NMR}$ $\left(100 \mathrm{MHz}, \mathrm{DMSOd}_{6}\right) \delta_{\mathrm{C}}: 171.7(\mathrm{C}=\mathrm{O}), 143.8,136.6,134.9,129.9(2 \times \mathrm{CH}), 129.6(2 \times \mathrm{CH}), 128.7(2 \times$ $\mathrm{CH}), 127.4,127.2(2 \times \mathrm{CH}), 56.4(\mathrm{CH}), 39.1\left(\mathrm{CH}_{2}\right), 30.3\left(\mathrm{CH}_{2}\right), 23.8\left(\mathrm{CH}_{2}\right), 23.5\left(\mathrm{CH}_{2}\right), 22.9\left(\mathrm{CH}_{2}\right), 18.6$ $\left(\mathrm{CH}_{3}\right)$ ppm. IR $(\mathrm{KBr}) v_{\max } / \mathrm{cm}^{-1}: 3367(\mathrm{~N}-\mathrm{H}), 3044(\mathrm{CH}$ aromatic $), 2922\left(\mathrm{C}-\mathrm{H}\right.$ of $\left.\mathrm{CH}_{2}\right), 1685(\mathrm{C}=\mathrm{O}$ of amide $), 1620(\mathrm{C}=\mathrm{C}), 1575(\mathrm{C}=\mathrm{N}), 1460\left(\mathrm{CH}_{2}\right.$ deformation $), 1377\left(\mathrm{SO}_{2}, 1^{\text {st }}\right.$ band $), 1178\left(\mathrm{SO}_{2}, 2^{\text {nd }}\right.$ band $)$, 1089 (C-N), 943 (=C-H), 725 (Ar-H). $\lambda_{\max } / \mathrm{nm}\left(\log \varepsilon_{\max }\right): 212$ (4.87), 548 (4.57), 575 (4.53), 593 (4.54).

\section{Synthesis of $\boldsymbol{N}$-cyclohexyl-3-phenyl-2-(phenylsulfonamido)propanamide, $9 \mathrm{i}$}

Reactive intermediate $8(3.30 \mathrm{mmol}, 1.00 \mathrm{~g})$ reacted with cyclohexylamine $(3.30 \mathrm{mmol}, 0.33 \mathrm{~g})$ to afford $\mathrm{N}$-cyclohexyl-3-phenyl-2-(phenylsulfonamido)propanamide, 9i in $63 \%$ yield. ${ }^{1} \mathrm{H}-\mathrm{NMR}(400 \mathrm{MHz}$, $\left.\mathrm{DMSOd}_{6}\right) \delta_{\mathrm{H}}$ : 7.62-7.59 (d, $\left.J=8.68 \mathrm{~Hz}, 2 \mathrm{H}, \mathrm{Ar}-\mathrm{H}\right), 7.25-7.21(\mathrm{~m}, 5 \mathrm{H}, \mathrm{Ar}-\mathrm{H}), 7.11-7.08$ (m, 3H, Ar-H), $5.88-5.86(\mathrm{~d}, J=8.16 \mathrm{~Hz}, 1 \mathrm{H}, \mathrm{NH}-\mathrm{CH}), 4.30-4.26(\mathrm{~m}, 1 \mathrm{H}, \mathrm{CH}), 4.21-4.18$ (quintet, $J=5.68 \mathrm{~Hz}, 1 \mathrm{H}$, $\left.\underline{\mathrm{CH}}\left(\mathrm{CH}_{2}\right)_{2}\right) 3.44-3.42\left(\mathrm{t}, J=3.68 \mathrm{~Hz}, 2 \mathrm{H}, \underline{\mathrm{NCH}_{2}} \mathrm{CH}_{2}\right), 3.12-3.08\left(\mathrm{dd}, J_{1}=5.48 \mathrm{~Hz}, J_{2}=20.00 \mathrm{~Hz}, 1 \mathrm{H}, \mathrm{CH}\right.$ of $\left.\mathrm{CH}_{2}\right), 3.03-2.98\left(\mathrm{dd}, J_{1}=6.40 \mathrm{~Hz}, J_{2}=20.00 \mathrm{~Hz}, 1 \mathrm{H}, \mathrm{CH}\right.$ of $\left.\mathrm{CH}_{2}\right), 1.61-1.58\left(\mathrm{~m}, 4 \mathrm{H}, 2 \times \mathrm{CH}_{2}\right), 1.49-$ $1.46\left(\mathrm{~m}, 4 \mathrm{H}, 2 \times \mathrm{CH}_{2}\right), 1.20-1.17\left(\mathrm{~m}, 2 \mathrm{H}, \mathrm{CH}_{2}\right) .{ }^{13} \mathrm{C}-\mathrm{NMR}\left(100 \mathrm{MHz}, \mathrm{DMSOd}_{6}\right) \delta_{\mathrm{C}}: 171.7(\mathrm{C}=\mathrm{O}), 143.8$, 136.6, 134.9, $129.9(2 \times \mathrm{CH}), 129.6(2 \times \mathrm{CH}), 128.7(2 \times \mathrm{CH}), 127.4,127.2(2 \times \mathrm{CH}), 56.4(\mathrm{CH}), 39.1$ $\left(\mathrm{CH}_{2}\right), 30.3\left(\mathrm{CH}_{2}\right), 23.8\left(\mathrm{CH}_{2}\right), 23.5\left(\mathrm{CH}_{2}\right), 22.9\left(\mathrm{CH}_{2}\right), 18.6\left(\mathrm{CH}_{3}\right) \mathrm{ppm}$. IR $(\mathrm{KBr}) v_{\max } / \mathrm{cm}^{-1}: 3365(\mathrm{~N}-\mathrm{H})$, $3040\left(\mathrm{CH}\right.$ aromatic), $2928\left(\mathrm{C}-\mathrm{H}\right.$ of $\left.\mathrm{CH}_{2}\right), 1685\left(\mathrm{C}=\mathrm{O}\right.$ of amide), $1620(\mathrm{C}=\mathrm{C}), 1575(\mathrm{C}=\mathrm{N}), 1460\left(\mathrm{CH}_{2}\right.$ deformation), 1377 ( $\mathrm{SO}_{2}, 1^{\text {st }}$ band), $1175\left(\mathrm{SO}_{2}, 2^{\text {nd }}\right.$ band), $1090(\mathrm{C}-\mathrm{N}), 943(=\mathrm{C}-\mathrm{H}), 721(\mathrm{Ar}-\mathrm{H}) . \lambda_{\max } / \mathrm{nm}$ $\left(\log \varepsilon_{\max }\right): 221$ (5.43), 260 (5.44), 578 (3.80), 584 (3.80).

\section{Synthesis of $\boldsymbol{N}$-hexadecyl-3-phenyl-2-(phenylsulfonamido)propanamide, $9 \mathbf{j}$}

Reactive intermediate $8(3.30 \mathrm{mmol}, 1.00 \mathrm{~g})$ reacted with hexadecylamine (3.30 mmol, $0.79 \mathrm{~g})$ to afford $N$ hexadecyl-3-phenyl-2-(phenylsulfonamido)propanamide, 9j in $91 \%$ yield. ${ }^{1} \mathrm{H}-\mathrm{NMR}\left(400 \mathrm{MHz}, \mathrm{DMSOd}_{6}\right.$ ) $\delta_{\mathrm{H}}$ : 7.61-7.59 (d, $\left.J=8.28 \mathrm{~Hz}, 2 \mathrm{H}, \mathrm{Ar}-\mathrm{H}\right), 7.24-7.21(\mathrm{~m}, 5 \mathrm{H}, \mathrm{Ar}-\mathrm{H}), 7.10-7.08$ (m, 3H, Ar-H), 5.89-5.87 (d, $J=8.88 \mathrm{~Hz}, 1 \mathrm{H}, \underline{\mathrm{NH}}-\mathrm{CH}), 4.30-4.26(\mathrm{~m}, 1 \mathrm{H}, \mathrm{CH}), 3.46-3.45\left(\mathrm{t}, J=3.06 \mathrm{~Hz}, 2 \mathrm{H}, \mathrm{NCH}_{2} \mathrm{CH}_{2}\right), 3.12-3.08$ $\left(\mathrm{dd}, J_{1}=5.48 \mathrm{~Hz}, J_{2}=20.00 \mathrm{~Hz}, 1 \mathrm{H}, \mathrm{CH}\right.$ of $\left.\mathrm{CH}_{2}\right), 3.03-2.98\left(\mathrm{dd}, J_{1}=6.40 \mathrm{~Hz}, J_{2}=20.00 \mathrm{~Hz}, 1 \mathrm{H}, \mathrm{CH}\right.$ of $\left.\mathrm{CH}_{2}\right), 1.54-1.51\left(\mathrm{~m}, 2 \mathrm{H}, \mathrm{CH}_{2}\right), 1.46-1.1 .42\left(\mathrm{~m}, 2 \mathrm{H}, \mathrm{CH}_{2}\right), 1.29-1.20\left(\mathrm{~m}, 24 \mathrm{H}, 12 \times \mathrm{CH}_{2}\right), 0.90-0.87(\mathrm{t}, J=$ $\left.4.22 \mathrm{~Hz}, 2 \mathrm{H}, \underline{\mathrm{CH}_{3}} \mathrm{CH}_{2}\right) .{ }^{13} \mathrm{C}-\mathrm{NMR}\left(100 \mathrm{MHz}, \mathrm{DMSOd}_{6}\right) \delta_{\mathrm{C}}: 171.7(\mathrm{C}=\mathrm{O}), 143.8,136.6,134.9,129.9(2 \times$ $\mathrm{CH}), 129.6(2 \times \mathrm{CH}), 128.7(2 \times \mathrm{CH}), 127.4,127.2(2 \times \mathrm{CH}), 56.4(\mathrm{CH}), 39.9\left(\mathrm{CH}_{2}\right), 39.1\left(\mathrm{CH}_{2}\right), 30.3$ $\left(\mathrm{CH}_{2}\right), 29.7\left(8 \times \mathrm{CH}_{2}\right), 29.1\left(2 \times \mathrm{CH}_{2}\right), 26.7\left(\mathrm{CH}_{2}\right), 23.5\left(\mathrm{CH}_{2}\right), 22.9\left(\mathrm{CH}_{2}\right), 15.9\left(\mathrm{CH}_{3}\right)$ ppm. IR $(\mathrm{KBr})$ $v_{\max } / \mathrm{cm}^{-1}: 3367(\mathrm{~N}-\mathrm{H}), 3044(\mathrm{CH}$ aromatic), $2922(\mathrm{CH}$ aliphatic $), 2848(\mathrm{CH}$ aliphatic), $1687(\mathrm{C}=\mathrm{O}$ of amide $), 1615(\mathrm{C}=\mathrm{C}), 1575(\mathrm{C}=\mathrm{N}), 1460\left(\mathrm{CH}_{2}\right.$ deformation $), 1376\left(\mathrm{SO}_{2}, 1^{\text {st }}\right.$ band $), 1175\left(\mathrm{SO}_{2}, 2^{\text {nd }}\right.$ band $)$, 1093 (C-N), 943 (=C-H), 725 (Ar-H). $\lambda_{\max } / \mathrm{nm}\left(\log \varepsilon_{\max }\right): 212$ (5.12), 265 (5.23), 378 (4.89), 545 (3.80).

\section{RESULTS AND DISCUSSION}

In the continuation of the research endeavor to design and derived more bioactive sulfonamide frameworks, ${ }^{12,29}$ we have herein reported the synthesis of functionalized carboxamide-based sulfonamide derivatives via a facile synthetic route. Synthesis of the precursor $\mathbf{7}$, reactive intermediate $\mathbf{8}$ and the targeted 
RASĀYAN J. Chem.

Vol. 10 | No. 4 |1402-1411 | October - December | 2017

carboxamide-based sulfonamide final products 9a-j were accomplished according to the steps illustrated in Schemes 1, 2 and 3 respectively. The synthesis began with the reaction of benzenesulfonyl chloride with L-phenyl alanine in the presence of sodium carbonate solution at ambient temperature for $10 \mathrm{~h}$ to afford 3phenyl-2-(phenyl sulfonamido) propanoic acid, 7 (Scheme 1). This was achieved in excellent yield via our earlier reported method. ${ }^{29}$ Secondly, the precursor 1 was esterified with absolute ethanol for $2 \mathrm{~h}$ in the presence of a mineral acid catalyst to afford ethyl 3-phenyl-2-(phenyl sulfonamide)propanoate, 8 (Scheme 2). The reaction of reactive intermediate sulfonamide $\mathbf{8}$ with aniline derivatives a-c via refluxing in the presence of ethanol afforded compounds $\mathbf{9 a - c}$, while the treatment of the compound $\mathbf{8}$ with $\alpha$-naphthylamine d furnished compound 9d. When the reactive sulfonamide $\mathbf{8}$ was heated under reflux in the presence of hydrazine hydrate e, 2,4-dinitrophenylhydrazine $\mathbf{f}$ and semicarbazide $\mathbf{g}$, the corresponding sulfonamidebased hydrazine carboxamide 9e-g were obtained in varying yields as shown in Table 1. Finally, the thermal reaction of $\mathbf{8}$ with three aliphatic amines $n$-pentylamine $\mathbf{h}$, cyclohexylamine $\mathbf{i}$ and hexadecylamine $\mathbf{j}$, over $2 \mathrm{~h}$ of refluxing provided a convenient route to access the corresponding sulfonamides $\mathbf{9 h} \mathbf{- j}$. The synthetic route for accessing all the final titled products 9a-j is as shown in Scheme 3.<smiles>O=C(O)C(Cc1ccccc1)NS(=O)(=O)c1ccccc1</smiles>

Scheme-1: Pathway for the synthesis of 3-phenyl-2-(phenyl sulfonamide)propanoic acid, 7<smiles>[Z]OC(=O)C(Cc1ccccc1)NS(=O)(=O)c1ccccc1</smiles><smiles>CCOC(=O)C(Cc1ccccc1)NS(=O)(=O)c1ccccc1</smiles>

Scheme-2: Pathway for the synthesis of ethyl 3-phenyl-2-(phenyl sulfonamide)propanoate, 8<smiles>[R]NC(=O)C(Cc1ccccc1)NS(=O)(=O)c1ccccc1</smiles>

9h: $\mathrm{R}=n$-pentyl

9i: $R=$ cyclohexyl 9j: $R=$ hexadecyl

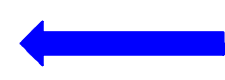<smiles>CCOC(=O)C(Cc1ccccc1)NS(=O)(=O)c1ccccc1</smiles>

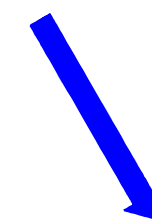

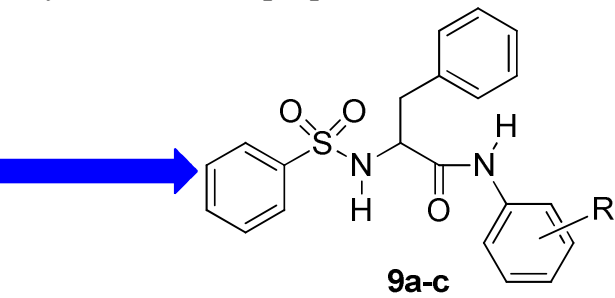

9a: $\mathrm{R}=\mathrm{H}$

9b: $\mathrm{R}=3-\mathrm{NO}_{2}$ 9e: $\mathrm{R}=\mathrm{H}$ 9f: $R=2,4$-dinitrophenyl

9g: $\mathrm{R}=\mathrm{O}=\mathrm{C}-\mathrm{NH}_{2}$<smiles>[R]NNC(=O)C(Cc1ccccc1)N(NS(=O)(=O)c1ccccc1)S(=O)(=O)[O-]</smiles>

Scheme-3: Synthetic route to $N$-substituted-3-phenyl-2-(phenylsulfonamido)propanamide, $9 \mathrm{a}-\mathrm{j}$ 
RASĀYAN $J$. Chem.

Vol. 10 | No. 4 |1402-1411 | October - December | 2017

The result of the physicochemical parameter which includes molecular formula, melting point, yield, $\mathrm{R}_{\mathrm{f}}$, and color and elemental analysis was herein reported (Table-1). The final products' molecular formula varied from 305.35 for compound $9 \mathbf{a}$ to 528.79 for $\mathbf{9 j}$. All the synthesized compounds had definite melting points except compound $9 \mathrm{~h}$ alone which was not determined because of its semi-solid nature at room temperature. The melting point of all the compounds was high, with $\mathbf{9 b}$ having lowest at the value of 173$175^{\circ} \mathrm{C}$ and 7 having the highest value of $296-298^{\circ} \mathrm{C}$. These high melting point values might be attributable to the amide bond formation and the tendency for hydrogen bond existence therein. The percentage yields of targeted compounds ranged from good to excellent in nature. Coincidentally, the first (precursor 7) and the last compound (sulfonamide $\mathbf{9 j}$ ) had the same yield of $91 \%$ which was the highest yield recorded herein while targeted compound 9e had the lowest yield (55\%) among the series. The Thin Layer Chromatography (TLC) was used to monitor the progress and completion of the reaction as well as to ascertain the purity level of the compounds synthesized. The $\mathrm{R}_{\mathrm{f}}$ calculated from the TLC varied from 0.44 for precursor 7 to 0.87 for sulfonamide $9 d$. Although, three different solvent ratio combination were used as eluents, the precursor 7 exhibiting lowest $\mathrm{R}_{\mathrm{f}}$ was an indication of high polarity due to the presence of $\mathrm{COOH}$ in $\mathbf{7}$. This further corroborated the highest melting point observed in precursor 7 among all the compound synthesized herein. This behavior in precursor $7 \mathrm{might}$ be as a result of the existence of stronger intramolecular hydrogen bonding in $\mathrm{COOH}$ than in the amide formed later after conversion to $\mathbf{9 a - j}$.

Table-1: Physicochemical parameter for the synthesized compounds $9 \mathrm{a}-\mathrm{j}$ and precursors

\begin{tabular}{|c|c|c|c|c|c|c|c|c|}
\hline Code & $\begin{array}{l}\text { Mol. Formular } \\
\text { (Mol. Weight) }\end{array}$ & M. Pt $\left({ }^{\circ} \mathrm{C}\right)$ & $\begin{array}{l}\text { Yield } \\
(\%)\end{array}$ & $\mathrm{Rf}$ & Colour & \multicolumn{3}{|c|}{$\begin{array}{l}\text { Elemental Analysis } \\
\% \text { Calcd. (\%Found) }\end{array}$} \\
\hline 7 & $\begin{array}{l}\mathrm{C}_{15} \mathrm{H}_{15} \mathrm{NO}_{4} \mathrm{~S} \\
(305.35)\end{array}$ & $296-298$ & 91 & $0.44^{\mathrm{y}}$ & $\begin{array}{l}\text { offwhite } \\
\text { powder }\end{array}$ & $\begin{array}{l}59.00 \\
(58.82) \\
\end{array}$ & $\begin{array}{l}4.95 \\
(5.01)\end{array}$ & $\begin{array}{l}4.59 \\
(4.68)\end{array}$ \\
\hline 8 & $\begin{array}{l}\mathrm{C}_{17} \mathrm{H}_{19} \mathrm{NO}_{4} \mathrm{~S} \\
(333.40)\end{array}$ & $241-243$ & 66 & $0.53^{x}$ & $\begin{array}{l}\text { White } \\
\text { pellet }\end{array}$ & $\begin{array}{l}61.24 \\
(61.41)\end{array}$ & $\begin{array}{l}5.74 \\
(5.92)\end{array}$ & $\begin{array}{l}4.20 \\
(4.39)\end{array}$ \\
\hline $9 \mathrm{a}$ & $\begin{array}{l}\mathrm{C}_{21} \mathrm{H}_{20} \mathrm{~N}_{2} \mathrm{O}_{3} \mathrm{~S} \\
(380.46)\end{array}$ & $219-221$ & 86 & $0.80^{x}$ & $\begin{array}{l}\text { White } \\
\text { powder }\end{array}$ & $\begin{array}{l}66.29 \\
(66.45)\end{array}$ & $\begin{array}{l}5.30 \\
(5.35)\end{array}$ & $\begin{array}{l}7.36 \\
(7.55)\end{array}$ \\
\hline $9 b$ & $\begin{array}{l}\mathrm{C}_{21} \mathrm{H}_{19} \mathrm{~N}_{3} \mathrm{O}_{5} \mathrm{~S} \\
(425.46)\end{array}$ & $173-175$ & 87 & $0.80^{y}$ & $\begin{array}{l}\text { Yellow } \\
\text { pellet }\end{array}$ & $\begin{array}{l}59.28 \\
(59.47)\end{array}$ & $\begin{array}{l}4.50 \\
(4.69)\end{array}$ & $\begin{array}{l}9.88 \\
(10.04)\end{array}$ \\
\hline $9 c$ & $\begin{array}{l}\mathrm{C}_{21} \mathrm{H}_{19} \mathrm{~N}_{2} \mathrm{O}_{3} \mathrm{SCl} \\
(414.91)\end{array}$ & $209-212$ & 74 & $0.82^{z}$ & $\begin{array}{l}\text { White } \\
\text { powder }\end{array}$ & $\begin{array}{l}60.79 \\
(60.95)\end{array}$ & $\begin{array}{l}4.62 \\
(4.45)\end{array}$ & $\begin{array}{l}6.75 \\
(6.83)\end{array}$ \\
\hline $9 \mathrm{~d}$ & $\begin{array}{l}\mathrm{C}_{25} \mathrm{H}_{22} \mathrm{~N}_{2} \mathrm{O}_{3} \mathrm{~S} \\
(430.52)\end{array}$ & $191-192$ & 65 & $0.87^{\mathrm{z}}$ & $\begin{array}{l}\text { Lilac } \\
\text { powder }\end{array}$ & $\begin{array}{l}69.75 \\
(69.91)\end{array}$ & $\begin{array}{l}5.15 \\
(4.98)\end{array}$ & $\begin{array}{l}\begin{array}{l}6.51 \\
(6.70)\end{array} \\
\end{array}$ \\
\hline $9 \mathrm{e}$ & $\begin{array}{l}\mathrm{C}_{15} \mathrm{H}_{17} \mathrm{~N}_{3} \mathrm{O}_{3} \mathrm{~S} \\
(319.38)\end{array}$ & $269-272$ & 54 & $0.64^{\mathrm{y}}$ & $\begin{array}{l}\text { White } \\
\text { powder }\end{array}$ & $\begin{array}{l}56.41 \\
(56.25) \\
\end{array}$ & $\begin{array}{l}5.37 \\
(5.52)\end{array}$ & $\begin{array}{l}13.16 \\
(12.97)\end{array}$ \\
\hline $9 \mathrm{f}$ & $\begin{array}{l}\mathrm{C}_{21} \mathrm{H}_{19} \mathrm{~N}_{5} \mathrm{O}_{7} \mathrm{~S} \\
(485.47)\end{array}$ & 188-190 & 82 & $0.69^{\mathrm{y}}$ & $\begin{array}{l}\text { Red } \\
\text { powder }\end{array}$ & $\begin{array}{l}51.95 \\
(52.09) \\
\end{array}$ & $\begin{array}{l}3.94 \\
(4.04)\end{array}$ & $\begin{array}{l}14.43 \\
(14.60)\end{array}$ \\
\hline $9 \mathrm{~g}$ & $\begin{array}{l}\mathrm{C}_{16} \mathrm{H}_{18} \mathrm{~N}_{4} \mathrm{O}_{4} \mathrm{~S} \\
(362.40)\end{array}$ & $208-210$ & 55 & $0.84^{x}$ & $\begin{array}{l}\text { Brown } \\
\text { crystal }\end{array}$ & $\begin{array}{l}53.03 \\
(52.91) \\
\end{array}$ & $\begin{array}{l}5.01 \\
(4.93) \\
\end{array}$ & $\begin{array}{l}15.46 \\
(15.63)\end{array}$ \\
\hline $9 \mathrm{~h}$ & $\begin{array}{l}\mathrm{C}_{20} \mathrm{H}_{26} \mathrm{~N}_{2} \mathrm{O}_{3} \mathrm{~S} \\
(374.50)\end{array}$ & Semi solid & 89 & $0.48^{x}$ & $\begin{array}{l}\text { Slurry } \\
\text { cream }\end{array}$ & $\begin{array}{l}64.14 \\
(63.99)\end{array}$ & $\begin{array}{l}7.00 \\
(6.85)\end{array}$ & $\begin{array}{l}7.48 \\
(7.64)\end{array}$ \\
\hline $9 \mathrm{i}$ & $\begin{array}{l}\mathrm{C}_{21} \mathrm{H}_{26} \mathrm{~N}_{2} \mathrm{O}_{3} \mathrm{~S} \\
(386.51)\end{array}$ & $244-246$ & 63 & $0.51^{x}$ & $\begin{array}{l}\text { Cream } \\
\text { powder }\end{array}$ & $\begin{array}{l}65.26 \\
(65.19)\end{array}$ & $\begin{array}{l}6.78 \\
(6.87)\end{array}$ & $\begin{array}{l}7.25 \\
(7.43)\end{array}$ \\
\hline $9 \mathrm{j}$ & $\begin{array}{l}\mathrm{C}_{31} \mathrm{H}_{48} \mathrm{~N}_{2} \mathrm{O}_{3} \mathrm{~S} \\
(528.79)\end{array}$ & $224-227$ & 91 & $0.58^{\mathrm{y}}$ & $\begin{array}{l}\text { Powder } \\
\text { ash }\end{array}$ & $\begin{array}{l}70.41 \\
(70.55)\end{array}$ & $\begin{array}{l}9.15 \\
(8.97)\end{array}$ & $\begin{array}{l}5.30 \\
(5.50)\end{array}$ \\
\hline
\end{tabular}

Solvent ratio $\mathrm{x}=(\mathrm{DCM} / \mathrm{Methanol} ; 9: 1), \mathrm{y}=(\mathrm{DCM} /$ Hexane; 9:1), $\mathrm{z}=(\mathrm{DCM} / \mathrm{Hexane} ; 8: 2)$

The spectroscopic characterization of the synthesized compounds was investigated using infrared, ultraviolet, ${ }^{1} \mathrm{H}$ and ${ }^{13} \mathrm{C}$ NMR. The ${ }^{1} \mathrm{H}$ NMR spectra of all synthesized compounds have been analyzed in DMSO- $d_{6}$ over the scan range of 0 to $13 \delta \mathrm{ppm}$. The spectroscopic result of compound 9a was given in detail as a representative of the final products $9 \mathbf{9}-\mathbf{j}$. The most downfield signal in $9 \mathbf{a}$ was $1 \mathrm{H}$ singlet of an amide at $\delta_{\mathrm{H}} 11.08 \mathrm{ppm}$. A doublet at $\delta_{\mathrm{H}} 7.62-7.60 \mathrm{ppm}$ was attributed to $2 \mathrm{H}$ aromatic linked to sulfonyl group and with a $J$ value of $8.34 \mathrm{~Hz}$, while the doublet assigned at $\delta_{\mathrm{H}} 7.45-7.43 \mathrm{ppm}$ was $2 \mathrm{H}$ aromatic linked to amide moieties and with a coupling constant of $8.00 \mathrm{~Hz}$. All other eleven protons of aromatic 
resonated as a $5 \mathrm{H}$ multiplet at $\delta_{\mathrm{H}} 7.27-7.24 \mathrm{ppm}, 3 \mathrm{H}$ multiplet at $\delta_{\mathrm{H}} 7.20-7.18 \mathrm{ppm}$ and $3 \mathrm{H}$ multiplet at $\delta_{\mathrm{H}}$ 7.15-7.12 ppm. These aromatic proton values were consistent with those earlier reported by Kushwaha $e t$ $a l .,{ }^{25}$ who synthesized and investigated some amide derivatives for their biological activity. The proton of $\mathrm{NH}$ signal linked $\mathrm{CH}$ resonated as a doublet at $\delta_{\mathrm{H}} 5.88-5.86 \mathrm{ppm}$, while that of its $\mathrm{CH}$ neighbor was noticed as a multiplet at $\delta_{\mathrm{H}} 4.31-4.25 \mathrm{ppm}$. This chemical shift in $\mathrm{CH}$ was due to deshielding effect as an inserted $\mathrm{CH}$ between sulfonamide and amide functionalities. The most upfield signal was that of $\mathrm{CH}_{2}$ linked to benzene and they resonated separately as a $1 \mathrm{H}$ doublet of doublet each at $\delta_{\mathrm{H}} 3.13-3.09 \mathrm{ppm}$ and 3.04-2.99 ppm respectively. The ${ }^{13} \mathrm{C}$ NMR spectra of all the synthesized compounds have been analyzed in DMSO$d_{6}$ over the scan range of 0 to $200 \mathrm{ppm}$. The ${ }^{13} \mathrm{C}-\mathrm{NMR}$ spectrum was run at $100 \mathrm{MHz}$ using DMSO- $d_{6}$ and compound 9a was observed to have its most downfield signal at $171.3 \mathrm{ppm}$ which was for carbonyl of the amide. Eighteen aromatic carbon were found from $145.1 \mathrm{ppm}$ to $115.2 \mathrm{ppm}$ while methane carbon $(\mathrm{CH})$ was found at $56.5 \mathrm{ppm}$ in compound 9a. The least signal was that of methylene $\left(\mathrm{CH}_{2}\right)$ which resonated at $39.0 \mathrm{ppm}$. In the overall for targeted products $9 \mathbf{a}-\mathbf{j}$, the most deshielded carbon was found in $\mathbf{9 g}$ with signal at $172.9 \mathrm{ppm}$ which was due to the presence of carbonyl of amide and it fell within the range of the values earlier reported by Ajani et al., ${ }^{26}$ who synthesized and examined spectroscopic features of some bioactive sulfonamide containing motifs. The most shielded carbon atom among the final product was found in $\mathbf{9 j}$ with chemical shift of $18.5 \mathrm{ppm}$ which depicted the presence of $\mathrm{CH}_{3}$ attached to long aliphatic carbon chains. However, it is worthy to note that the chemical shift of $\mathrm{C}=\mathrm{O}$ of precursor $7\left(\delta_{\mathrm{c}} 178.5 \mathrm{ppm}\right)$ was higher than that of all the final products $9 \mathbf{a}-\mathbf{j}\left(\delta_{\mathrm{c}} 171.1-172.9 \mathrm{ppm}\right)$, which implied that there was an obvious synthetic modification on the $\mathrm{COOH}$ of precursor 7 to have led to the final product which was bearing $\mathrm{C}=\mathrm{O}$ of amide. The careful evaluation of infrared spectral feature of compound 9a revealed that the absorption frequency at $3415 \mathrm{~cm}^{-1}$ was for $\mathrm{N}-\mathrm{H}$ functionality and it was doubly confirmed by the presence of bending vibration bands at $1491 \mathrm{~cm}^{-1}$ and C-N functionality at $1073 \mathrm{~cm}^{-1}$. The absorption frequencies at $2930 \mathrm{~cm}^{-1}$ and $2850 \mathrm{~cm}^{-1}$ were for $\mathrm{C}-\mathrm{H}$ of aliphatic which was confirmed by the occurrence of $\mathrm{CH}_{2}$ deformation at $1459 \mathrm{~cm}^{-1}$ which were consistent with an earlier report. ${ }^{30}$ The absorption frequencies at $1685 \mathrm{~cm}^{-1}$ as well as $1610 \mathrm{~cm}^{-1}$ in compounds 9 a showed the presence carbonyl of amide and $\mathrm{C}=\mathrm{C}$ aromatic respectively. The two sulfonyl bands were present at $1377 \mathrm{~cm}^{-1}$ and $1140 \mathrm{~cm}^{-1}$. This fell within the range earlier reported by Ajani et al. ${ }^{12}$ for some bioactive sulfonamides. The UV spectrum of 9a was run in methanol and the wavelength $\lambda_{\max }$ was recorded in nanometer $(\mathrm{nm})$ alongside with the $\log \varepsilon_{\max }$. Generally, the electronic transition of UV-visible spectra of all synthesized compounds in methanol led to the $\lambda_{\max }$ values from 206 $\mathrm{nm}$ to $593 \mathrm{~nm}$. Specifically speaking for 9a, the first wavelength was observed at $207 \mathrm{~nm}\left(\log \varepsilon_{\max } 5.01\right)$ which was as a result of $\pi \rightarrow \pi^{*}$ transition peculiar to $\mathrm{C}=\mathrm{C}$ of the benzene ring. Other transitions were observed in the UV spectrum of 9a giving rise to $\lambda_{\max }$ values at $244 \mathrm{~nm}\left(\log \varepsilon_{\max } 5.47\right), 286 \mathrm{~nm}\left(\log \varepsilon_{\max }\right.$ 5.11) and $583 \mathrm{~nm}\left(\log \varepsilon_{\max } 2.00\right)$. The bathochromic shift observed at the higher wavelengths between 244 $\mathrm{nm}$ to $583 \mathrm{~nm}$ was due to the occurrence of $\pi \rightarrow \mathrm{n}$ transition that was resulting from the presence of the chromophoric moieties and auxochromic group attached to the ring system. The delocalization of their lone pairs played a key role in the bathochromic shift occurrence experienced herein.

\section{CONCLUSION}

Based on the results, it can be concluded that the synthesis of the sulfonamide bearing diversified carboxamide was successfully achieved. The characterization carried out authenticated the structures of the targeted products as envisaged. The technique used herein was found to be efficient and cost-effective for the production of the series of new sulfonamide derivatives. The targeted compounds are good candidates for further study for antimicrobial investigation and could probably be the potential source of chemotherapeutic drugs.

\section{ACKNOWLEDGEMENT}

OOA is thankful to The World Academy of Sciences for the sponsorship of this project under the TWAS Research Grants Programme in Basic Sciences for Individual Scientists (Grant No. 14-069 RG/CHE/AF/AC_1).

\section{REFERENCES}

1. L. Graham and J. Patrick, J. Chimie Pharmaceutique. Paris: Deboek, 380 (2003). 
RASĀYAN $J$. Chem.

Vol. 10 | No. 4 |1402-1411 | October - December | 2017

2. I. Beronica, G. Malin, L. Mattias, K. Charlotte, L. Pallecchi, G.M. Rossolini and K. Goran, Int. J. Antimicrob. Agents, 25, 308 (2005).

3. S.F. Yang, C.F. Lin, C.J. Wu, K.K. Ng, A.Y.C. Lin and P.K.A. Hong, Water Res., 46, 1301(2012).

4. Z. Köksal, R. Kalin, Y. Camadan, H. Usanmaz, Z. Almaz, I. Gülçin, T. Gokcen, A.C. Gören and H. Ozdemir, Molecules, 22, 793 (2017).

5. G.A. Domagk, Clin. Infect. Dis., 8(1), 163 (1986).

6. D. Vicente and E. Perez-Trallero, Enfermedades Infecciosas y Microbiología Clínica, 28(2), 122 (2010).

7. Z. Chen, W. Xu, K. Liu, S. Yang, H. Fan, P.S. Bhadury, D.Y. Huang and Y. Zhang, Molecules, 15(12), 9046 (2010).

8. H. Kourlas and S. Morey, J. Pharm. Pract., 20(5), 399 (2007).

9. F.A. Fehintola, A.A. Adedeji, E. Tambo, B.B. Fateye, T.C. Happi and A. Sowunmi, Clin. Drug Invest., 24(3), 149 (2004).

10. E.H. Moylett, S.E. Pacheco, B.A. Brown-Elliot, T.R. Perry, E.S. Buescher, M.C. Birmingham, J.J. Schentag, J.F. Gimbel, A. Apodaca, M.A. Schwartz, R.M. Rakita and R.J. Wallace, Clin. Infect. Dis., 36(3), 313 (2003).

11. A.K. Gadad, C.S. Mahajanshetti, S. Nimbalkar, and A. Raichurkar, Eur. J. Med. Chem., 35, 853 (2000).

12. O.O. Ajani, O.B. Familoni, F. Wu, J.O. Echeme, and Z. Sujiang, Int. J. Med. Chem., 2012, (2012). Article ID 367815, 13 pages. doi:10.1155/2012/367815.

13. P.T. Evanthia, F.S. Victoria, and N.P. Ioannis, Curr. Pharm. Anal., 6(3), 198 (2010).

14. P. Purushottamachar, A. Khandelwal, T.S. Vasaitis, R.D. Bruno, L.K. Gediya, and V.C.O. Njar, Bioorg. Med. Chem., 16(7), 3519 (2008).

15. G.M. Fisher, S. Bua, S.D. Prete, M.S.J. Arnold, C. Capasso, C.T. Supuran K.T. Andrews, and S.A. Poulsen, Int. J. Parasitol. Drugs Drug Resist., 7(1), 61 (2017).

16. S. Hessainia, F. Bouchareb, H. Cheloufi, M. Berredjem, H. Berredjem, I. Becheker, N.E. Aouf, Rasayan J. Chem., 6(3), 175 (2013).

17. N.P. Lad, S. Kulkarni, R. Sharma, M. Mascarenhas, M.R. Kulkarni, and S.S. Pandit, Eur. J. Med. Chem., 126, 870 (2017).

18. R. Ulus, B.Z. Kurt, I. Gazioğlu, and M. Kaya, Bioorg. Chem., 70, 245 (2017).

19. K.M. Naidu, H.N. Nagesh, M. Singh, D. Sriram, P. Yogeeswari, K. Venkata and G.C. Sekhar, Eur. J. Med. Chem., 92, 415 (2015).

20. R. Muthukumaran, B. Sangeetha, R. Amutha and P.P. Mathur, Computer-Aided Drug Des., 8(1), 70 (2012).

21. L. Sun, Y. Wu, Y. Liu, X. Chen and L. Hu, Bioorg. Med. Chem. Lett., 27(2), 261 (2017).

22. B. Masereel, S. Rolin, F. Abbate, A. Scozzafava and C.T. Supuran, J. Med. Chem., 45(2), 312 (2002).

23. P. Zajdel, K. Marciniec, A. Maślankiewicz, K. Grychowska, G. Satała, B. Duszyńska, T. Lenda, A. Siwek, G. Nowak, A. Partyka, D. Wróbel, M. Jastrzębska-Więsek, A.J. Bojarski, A. Wesołowska and M. Pawłowski, Eur. J. Med. Chem., 60, 42 (2013).

24. M. Stumvoll, B.J. Goldstein and T.W. van Haeften, Lancet, 365, 1333 (2005).

25. S.B. Sawant, D.V.N.S. Rao, C. Nageswarrao, P.P. Reddy, R. Agarwal and R. Sharma, Rasayan J. Chem., 8(4), 527 (2015).

26. O.O. Ajani, D.V. Aderohunmu, J.D. Udonne, C.O. Ehi-Eromosele, J.A.O. Olugbuyiro, C.O. Ajanaku and O.Y. Audu, Curr. Res. Chem., 8(1-3), 10 (2016).

27. K.N. Puri and G.V. Korpe, Rasayan J. Chem., 9(1), 52 (2016).

28. A.R. Katritzky C. Cai and S.K. Singh, J. Org. Chem., 71, 3375 (2006).

29. O.O. Ajani, O.B. Familoni, J.O. Echeme, F. Wu, and Z. Sujiang. Am. Chem. Sci. J., 3(1), 34 (2013).

30. O.O. Ajani, O.B. Familoni, F. Wu, J.O. Echeme, and Z. Sujiang. Int. J. Drug Des. Disc., 4(1), 983 (2013).

[RJC-1816/2017] 